\title{
Evaluation of genetic algorithm based solar tracking system for photovoltaic panels
}

\begin{abstract}
The maximum power supplied by a photovoltaic (PV) panels system change over time. It depends on environmental factors such as the solar irradiation and the temperature of these panels. The average solar energy harvested by the conventional solar panels during the course of the day, is not always maximized. This is due to the static placement of the panel which limits their area of exposure to the sun. In practice, there are three possible approaches for maximizing the solar power extraction in medium and large scale PV systems are sun tracking, maximum power point (MPP) tracking or combination of both. In this paper, a genetic algorithm (GA) has been proposed utilizing sun tracking approaches to maximize the performance of PV panels. Literature suggested that the PV panels could produce maximum power if the panels have angle of inclination zero degree to the sun position. This work evaluate the best combination of GA parameters to optimize a solar tracking system for PV panels in terms of azimuth angle and tilt angle. Simulation results demonstrated the ability of the proposed GA system to search for optimal panel positions in term of consistency and convergence properties. It also has proved the ability of the GA-solar to adapt to different environmental conditions and successfully track sun positions in finding the maximum power by precisely orienting the PV panels.
\end{abstract}

Keyword: Genetic algorithm; PV panels; Solar tracking 